\title{
NLTE spectral analysis of the intermediate helium-rich subdwarf B star CPD-20¹123*
}

\author{
L. Löbling ${ }^{1 \dagger}$ \\ ${ }^{1}$ Institute for Astronomy and Astrophysics, Kepler Center for Astro and Particle Physics, Eberhard Karls University, \\ Sand 1, 72076 Tübingen, Germany
}

Accepted 2020 June 3. Received 2020 May 29; in original form 2019 August 21

\begin{abstract}
Subdwarf B (sdB) stars are core helium-burning stars with stratified atmospheres. Their atmospheres are dominated by hydrogen $(\mathrm{H})$ while the helium $(\mathrm{He})$ and metal abundances are shaped by an interplay of gravitational settling and radiative levitation. However, a small fraction of these show spectra dominated by He i absorption lines. In between these groups of He-deficient and extreme He-rich sdBs, some are found to have intermediate surface He abundances. These objects are proposed to be young "normal" (He-deficient) sdBs for which the dynamical stratification of the atmosphere is still ongoing. We present an analysis of the optical spectrum of such an intermediate He-rich sdB, namely $\mathrm{CPD}-20^{\circ} 1123$, by means of non-local thermodynamic equilibrium (NLTE) stellar atmosphere models. It has a He-to-H number ratio of $\mathrm{He} / \mathrm{H}=0.13 \pm 0.05$ and its effective temperature of $T_{\text {eff }}=25500 \pm 1000 \mathrm{~K}$ together with a surface gravity of $\log \left(\mathrm{g} / \mathrm{cm} / \mathrm{s}^{2}\right)=5.3 \pm 0.3$ places the star close to the hightemperature edge until which it may be justified to use LTE model atmospheres. This work states a test of the Tübingen NLTE Model Atmosphere Package for this temperature regime. We present the first application of revised, elaborated model atoms of low ionization stages of light metals usable with this atmosphere code.
\end{abstract}

Key words: stars: subdwarfs - stars: abundances - stars: evolution - stars: atmospheres - stars: chemically peculiar - stars: individual: CPD-20 1123

\section{INTRODUCTION}

Subdwarf B (sdB) stars are located at the high-temperature end of the horizontal branch (HB) or have evolved off the $\mathrm{HB}$ and hence are stars with a central helium (He) burning region. They have thin hydrogen $(\mathrm{H})$ envelopes and in most cases, their atmospheres are He deficient due to gravitational settling. The abundances of metals are the result of the interaction of gravitational settling and radiative levitation (Heber 2016). However, a fraction of $5 \%$ (Ahmad \& Jeffery 2006) to $13 \%$ (Green et al. 1986) of the total subdwarf population show He-rich atmospheres. Among these Hesubdwarf stars (He-sds), a small number has intermediate $\mathrm{He}$ abundances between $5 \%$ and $80 \%$ by number (Naslim et al. 2010). These stars are regarded as transition objects evolving from a He-rich progenitor to a He-poor subdwarf star and, thus, are of particular interest (Heber 2016).

\footnotetext{
* Based on data products from observations made with ESO Telescopes at the La Silla Paranal Observatory under programme ID 086.D-0714(A).

† E-mail: loebling@astro.uni-tuebingen.de
}

CPD-20¹123 (Albus 1, Gill \& Kapteyn 1896; Vennes et al. 2007 ) is a blue star and one of the brightest known He-sdB stars. Based on photometric data only, Caballero \& Solano (2007) proposed that it might be a hot white dwarf (WD) similar to the DA-type WD G191-B2B or alternatively a hot subdwarf. Using low-resolution optical spectra, Vennes et al. (2007) could confirm the subdwarf classification and found a He enrichment. Using NLTE model atmospheres, they found $T_{\text {eff }}=19800 \pm 400 \mathrm{~K}, \log g=4.55 \pm 0.10$, and a He abundance of $\log \mathrm{He} / \mathrm{H}=0.15 \pm 0.15$. Naslim et al. (2012, hereafter N12) had access to high-resolution optical spectra and performed a comprehensive spectral analysis based on LTE model atmospheres. They determined element abundances for elements up to Fe and found $T_{\mathrm{eff}}=23500 \pm 500 \mathrm{~K}$, $\log g=4.9 \pm 0.1$, and a He abundance of $0.17 \pm 0.05$ based on LTE atmospheres. With a series of radial velocity measurements, they determined an orbital period of the binary to be $2.3 \mathrm{~d}$ and argued that the system is a close binary that underwent common-envelope evolution. Its intermediate He abundance can thus be an indicator for a young sdB star in which the diffusion driven stratification of the atmosphere is not yet in equilibrium but still ongoing. 
For the spectral analysis by means of model atmosphere techniques, a close correspondence between the calculated model and the stellar atmosphere is necessary. Compromises and simplifications need to be taken into consideration due to limitations on computational capacity and available atomic data. While it is highly recommended to use non-local thermodynamic equilibrium (NLTE) model atmospheres for hot white dwarfs and subdwarf O (sdO) stars, it may be justified to use LTE atmospheres for the analysis of sdB stars (Napiwotzki 1997). The departure from LTE should be small in this regime due to high densities in the atmosphere. However, CPD $-20^{\circ} 1123$ is located at the high- $T_{\text {eff }}$ border of the domain of LTE codes. This implies, that NLTE codes are not frequently used and tested for this regime. This work presents a NLTE analysis of the optical spectrum of CPD-20 1123 and states a test of the Tübingen Model Atmosphere Package (TMAP ${ }^{1}$, Werner et al. 2003, 2012) for this temperature and gravity regime. We describe the observations in Sect. 2 and introduce the model-atmosphere code as well as the revisions and updates that were done on the Tübingen Model Atom Database (TMAD, Rauch \& Deetjen 2003) in Sect. 3. The analysis is described in Sect. 4 and Sect. 5. The results are discussed in Sect. 6 and we end with our conclusions in Sect. 7.

\section{OBSERVATIONS}

We use high-resolution, high signal-to-noise observations for this analysis. The spectra were partly processed with a lowpass filter (Savitzky \& Golay 1964). All synthetic spectra shown in this paper are convolved with Gaussians to simulate the instruments' resolving power $R=\lambda / \Delta \lambda$.

Six optical spectra were obtained with the University College London Echelle Spectrograph (UCLES) at the AngloAustralian Telescope (AAT) on 2010-01-14 (3820 $\AA<\lambda<$ $5230 \AA$ A, grating with 31 lines $\mathrm{mm}^{-1}, R \approx 45000$ ) with a total exposure time of $9000 \mathrm{~s}$. Details about calibration and and post-processing are given by N12.

In addition, we used six high-resolution spectra taken with the Fiberfed Extended Range Optical Spectrograph (FEROS) at the MPG/ESO $2.2 \mathrm{~m}$ telescope $(3700 \AA<\lambda<$ $9200 \AA, R \approx 48000)$ on $2010-10-30(700 \mathrm{~s})$ and $2010-11-01$ $(2 \times 450 \mathrm{~s}, 2 \times 600 \mathrm{~s}$, and $900 \mathrm{~s})$. These were retrieved as raw data from the ESO science archive and reduced with the standard ESO MIDAS pipeline which does not perform a background subtraction.

\section{MODEL ATMOSPHERES AND ATOMIC DATA}

For our spectral analysis, we calculated a grid of stellaratmosphere models using TMAP. This code calculates chemically homogeneous NLTE models under the assumption of plane-parallel geometry in hydrostatic and radiative equilibrium. Level dissolution (pressure ionization) is considered following Hummer \& Mihalas (1988) and Hubeny et al. (1994) for all species. The H I line profiles were calculated using the line broadening tables of Tremblay \& Bergeron

1 http://astro.uni-tuebingen.de/ TMAP
(2009, extended tables of 2015, priv. comm.). To consider the line broadening of He i $\lambda \lambda 4026,4338,4471,4921 \AA$, tables of Barnard et al. (1969) and Barnard et al. (1974, only for He I $\lambda 4471 \AA$, for electron densities $10^{13}<n_{e} / \mathrm{cm}^{3}<10^{16}$ ) were used. For the lines He i $\lambda \lambda 4121,4438,4713,5017,5048 \AA$ we used broadening tables of Griem (1974) and for all other lines in the range $3732<\lambda<8997$ we used the tables of Beauchamp et al. (1997). The line profiles of He I $\lambda \lambda 4144,4438,8915 \AA$ and of all lines outside of the region mentioned above were calculated using an approximate formula for the quadratic Stark effect with parameters of Dimitrijević \& Sahal-Bréchot (1990). The lines He I $\lambda \lambda 3926,4009,4143 \AA$ feature in the Beauchamp et al. (1997) tables but the modeled line shapes do not agree with the observations. Most likely, our treatment of the physics of these lines is incomplete but since these lines are not used for any parameter determination, they do not affect the results. Line broadening tables for some $\mathrm{Al}$ II lines are available from Dimitrijević \& Sahal-Bréchot (1993) and used for the calculation of the line profiles. Broadening of all other Al lines due to the quadratic Stark effect is calculated using approximate formulae by Cowley $(1970,1971)$. Atomic data for the model calculations were obtained from TMAD. This database was enlarged and extended in the course of this analysis (Sect. 3.1). For the iron-group elements (IGEs) (Ca-Ni), we used Kurucz's line lists (Kurucz 1991, 2009) and constructed statistical model atoms using the Tübingen Iron-Group Opacity (TIRO) - WWW Interface.

\subsection{New and revised model atoms}

To reproduce the variety of metal lines found in the optical spectrum of $\mathrm{CPD}-20^{\circ} 1123$ (N12), we constructed new model atoms for aluminum, phosphorus, and chlorine and revised and substantially extended the low ionization stages (II-IV) of the fluorine, sulfur (only for spectrum synthesis), and argon model atoms.

The majority of level energies were retrieved from the National Standards and Technology Institute (NIST) Atomic Spectra Database $\left(\mathrm{ASD}^{2}\right)$. Oscillator strengths, photoionization crosssections, and additional energy levels were calculated in the framework of the Opacity Project $\left(\mathrm{OP}^{3}\right.$, Seaton 1987). Additional sources of data were Eriksson (1983) and Becker \& Butler (1989) for N II, and Wiese et al. (1969), Fuhr \& Wiese (1998), Bengtson (1968), Foster (1962), Varsavsky (1961), and Abou El-Maaref et al. (2012) for Cl II-IV. Higher ionization stages of the new model atoms have already been used for the analysis of hot white dwarfs (e.g., Rauch et al. 2017).

\section{ATMOSPHERIC PARAMETERS}

The model grid calculated for this analysis spans around the literature values for $T_{\text {eff }}, \log g$, and $\mathrm{He} / \mathrm{H}$ of $\mathrm{N} 12$ $\left(T_{\text {eff }}=22500(1000) 27500, \log g=4.3(0.2) 5.9\right.$, and $\mathrm{He} / \mathrm{H}=$ $0.1(0.1) 0.3)$ considering the opacities of 22 elements. The

\footnotetext{
2 https://physics.nist.gov/PhysRefData/ASD/levels_form. html

3 http://cdsweb.u-strasbg.fr/topbase/topbase.html
} 
statistics of the model atoms used are shown in Table A1 and the ionization fractions in Fig. A1. The initial metal abundances are taken from N12 while the abundances for the IGEs not yet analysed were scaled to Fe. For F, we adopted the solar value.

Like N12, we used models with a microturbuent velocity of $v_{t}=10 \mathrm{~km} \mathrm{~s}^{-1}$. The atmospheric parameters $T_{\text {eff }}$, $\log g$, and the $\mathrm{He} / \mathrm{H}$ ratio, were determined from a simultaneous fit of $\mathrm{H}$ and $\mathrm{He}$ lines. The line list of N12 has been extended by lines of Schindewolf et al. (2018). The final list is summarized in Table A2. The best fit yields $T_{\text {eff }}=25500 \pm 1000 \mathrm{~K}, \log g=5.3 \pm 0.3$, and $\mathrm{He} / \mathrm{H}=0.13 \pm 0.05$. These values differ from the parameters determined by N12 $\left(T_{\mathrm{eff}}=23500 \pm 500 \mathrm{~K}, \log g=4.9 \pm 0.1\right.$, and $\mathrm{He} / \mathrm{H}=0.17 \pm$ $0.05)$. They used a smaller set of Stark-broadenend He and $\mathrm{H}$ lines to derive the surface gravity. Figure 1 illustrates that these lines in the UCLES spectrum are not fit equally well by the synthetic spectra with a given $\log g$. By employing also the FEROS observations, we could enlarge the list of broad lines but a simultaneous fit of the data of the two instruments could not be achieved since the observations show significant deviations in the lines that are covered by both. Compared to the UCLES observation, the line cores in the synthetic spectra appear to be too deep. This problem was also seen for the LTE models of N12. Whereas the line cores in the FEROS observation are deeper and the synthetic spectra are too shallow. This may be an effect of different data reduction procedures. While the UCLES observations are background subtracted, the standard FEROS pipeline does no background subtraction. We speculate that background subtraction for the FEROS spectra would even increase the discrepancy. Our determination of the atmospheric parameters is, thus, entirely based on the UCLES spectrum. The FEROS observations are used for cross-check and for abundance determinations in wavelength ranges not covered by UCLES.

N12 used the ionization equilibria of SiII/III and S II/III to determine $T_{\text {eff }}$ and reported that they do not coincide in a single point but that SiII/III yields a higher temperature than SII/III. Figure 2 illustrates that this is seen in the same way in our NLTE analysis, although we find higher temperatures. The best $T_{\text {eff }}$ for the SiII/III lines is in very good agreement with the best fit from the $\mathrm{H}$ and He lines, while the $\mathrm{SII} / \mathrm{III}$ lines would require a $T_{\text {eff }}$ that is lower by approximately $1000 \mathrm{~K}$. A former NLTE analysis by Vennes et al. (2007) yielded lower values of $T_{\text {eff }}=19800 \pm 400 \mathrm{~K}$ and $\log g=4.55 \pm 0.1$ based on a lowresolution optical spectrum. The discrepancy might arise from the fact that they used the Balmer and He I line-profile fits for the determination of both, $T_{\text {eff }}$ and $\log g$ and the $\mathrm{H}_{\mathrm{I}}$ Stark-broadening tables of Lemke (1997) or might be an effect of the borders of the model grid and an inclusion of higher temperatures could have resulted in parameters similar to this paper. Most likely, the use of models containing $\mathrm{H}$ and $\mathrm{He}$ in contrast to models that consider opacities of 22 elements might have an effect on the final result. But still in the present analysis, the Balmer line fits (Figure 1) indicate missing opacity. The missing flux in the UV indicated by the GALEX observations (Figure A2) confirms this explanation.

\section{ABUNDANCES}

We adopted $T_{\text {eff }}=25500 \mathrm{~K}$ and $\log g=5.30$ for the abundance measurements. Based on the model grid with 22 elements (statistics given in Table A1), we performed line-formation calculations for each element individually. In these, the number of NLTE levels is enlarged and the temperature structure is kept fixed to calculate the occupation numbers for the levels. The abundances were derived by line-profile fits and are affected by typical errors of 0.3 dex including the statistical error from the use of several lines and the error propagation from our uncertainties in $T_{\text {eff }}$ and $\log g$. For some elements, no line could be identified in the observation. In these cases, we derived upper abundance limits by reducing the abundance until the most prominent computed lines become undetectable within the noise of the spectrum. The resulting abundances are summarized in Table A3 and Fig. 3. The whole UCLES spectrum compared to our final model is shown in Fig. 4.

\section{$5.1 \quad$ Light metals}

For our abundance measurements, we used the same lines as N12. We could not identify any line of F but included it in the atmosphere calculation with solar abundances to take its opacity into account. No lines of $\mathrm{Na}$ are present in the observations but an upper limit of $[\mathrm{Na}] \leq-1.26$ (with $[\mathrm{X}]=$ $\log ($ mass fraction/solar mass fraction) $)$ can be derived based on Na I $\lambda \lambda 5148.8,5153.4 \AA$ in the FEROS range. The Ne abundance of $[\mathrm{Ne}]=0.23$ is determined based on $\mathrm{Ne}$ I $\lambda 6402.2 \AA$. The individual abundance for $\mathrm{Al}$ In $\lambda \lambda 4479.9,4480.0 \AA$ is omitted from the mean since it yields a significantly lower abundance compared to the result for all other analyzed lines of $\mathrm{Al}$ (Fig. A3). This is contrary to the result of N12 who found the largest individual line abundance for these lines. We omitted the line P III $\lambda 4059.3 \AA$ from our analysis due to the non-detection of $\mathrm{P}_{\text {III }} \lambda 4080.1 \AA$ which belongs to the same multiplet (Fig. A3). We include P III $\lambda 4246.7 \AA$ which was not considered by N12. For Cl, they report a significantly higher abundance for $\mathrm{Cl}_{\text {II }} \lambda 4896.8 \AA$ compared to $\mathrm{Cl}_{\text {II }} \lambda \lambda 4794.6,4810.1 \AA$ and, thus, omitted the first line. We see overall higher abundances for each line but find the lowest value for $\mathrm{Cl}_{\text {II }} \lambda 4896.8 \AA$ (Table 1 , Fig. 5). This trend of higher abundances in our analysis compared to the results of N12 is seen for all light metals with the only exception of Ar.

\subsection{Iron-group elements}

We determine an abundance of $[\mathrm{Ca}]=0.67$ based on Ca II $\lambda \lambda 3933.7,3968.5 \AA$. The Fe abundance of $[\mathrm{Fe}]=-0.24$ is determined by analyzing 14 lines of Fe III (Table 1). This element is the only metal, for which we found a lower abundance compared to the values found in the LTE analysis of N12. This can already be seen when comparing the abundances for the four Fe III lines considered by them (Table 1, Fig. A4). Again, we find, that there is not only an offset between the different analyses but that the discrepancies are different from line to line. For all other IGE, we could not identify any line but derived upper abundance limits. The values for $\mathrm{Ti}$ and $\mathrm{V}$ are very close to the ones found by N12 whereas our results for Sc and Cr are higher than theirs. Mn, 


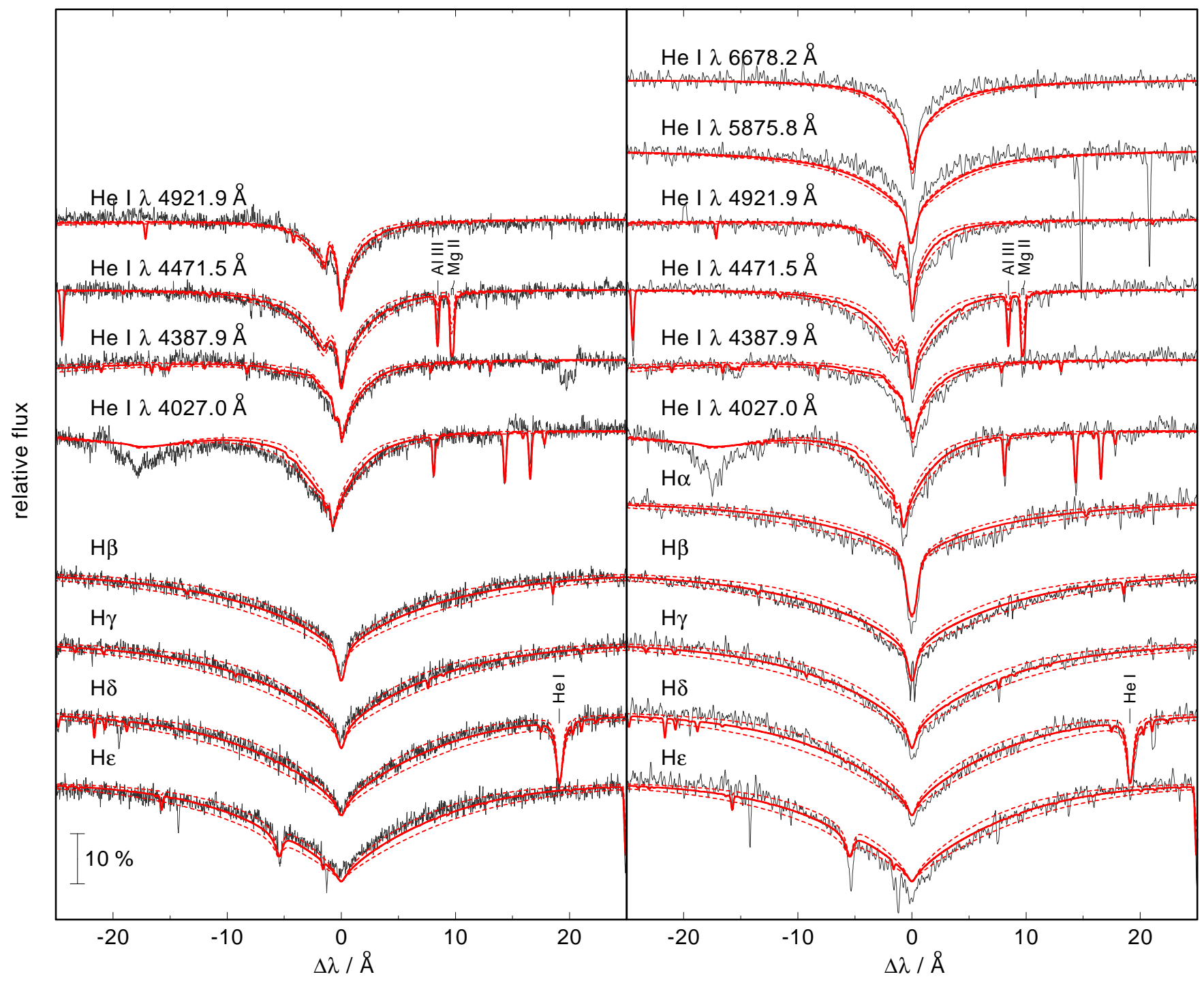

Figure 1. Synthetic spectra calculated with $T_{\mathrm{eff}}=25500 \mathrm{~K}$ and $\log g=5.3$ (red, solid) and $\Delta \log g=0.4$ (red, dotted), compared with the UCLES (left) and FEROS (right) observations of He I $\lambda$ 4027.0, 4387.9, 4471.5, 4921.9, 5875.8, 6678.2 $\AA$, $\mathrm{H} \alpha, \mathrm{H} \beta, \mathrm{H} \gamma, \mathrm{H} \delta$, and $\mathrm{H} \epsilon$. The vertical bar indicates $10 \%$ of the continuum flux.

Co, and Ni were not analyzed before and we use the computed lines Mn III $\lambda 4292.9 \AA$, Co III $\lambda \lambda 3932.9,3955.1 \AA$, and $\mathrm{Ni}$ iII $\lambda \lambda 4362.8,4363.7,4365.4 \AA$ to determine upper abundance limits.

\section{DISCUSSION}

\subsection{Departures from LTE}

The aim of this work is to enlarge and update TMAD for low ionization stages of light metals and apply the new model atoms in an analysis of an object at the border of the regimes of NLTE and LTE codes, that has already been analyzed comprehensively by means of LTE. The departure coefficients (ratio of NLTE and LTE occupation numbers) are a measure for the importance of NLTE effects. Of special interest are the departure coefficients of the new model atoms. Figure 6 shows the departure coefficients for the lev- els of $\mathrm{Al}$ II, $\mathrm{Al}$ III, $\mathrm{P}$ III, and $\mathrm{Cl}_{\text {II }}$ corresponding to the lines in Fig. A3, and 5. In the line-forming region, all these coefficients are below unity. The speculation that these lower NLTE occupation numbers compared to LTE are responsible for the higher light metal abundances could be ruled out by a LTE model produced with TMAP. This can be achieved in TMAP by artificially increasing the collisional rates in the calculation and thus forcing the departure coefficients to unity. Fig. 7 shows the temperature structure of the two different models. Although the ratio $T_{\text {NLTE }} / T_{\text {LTE }}$ is close to unity, a slightly lower $T_{\mathrm{NLTE}}$ is visible in the inner part of the line-forming region while the outermost part shows a higher $T_{\text {NLTE }}$. The difference in emergent flux between the TMAP NLTE and LTE model does not exceed $2 \%$, which can by far not explain the abundance discrepancies between the analysis of N12 and this work. It needs to be questioned whether the differences arise from different codes or are an effect of the elemental composition of the model atmosphere 


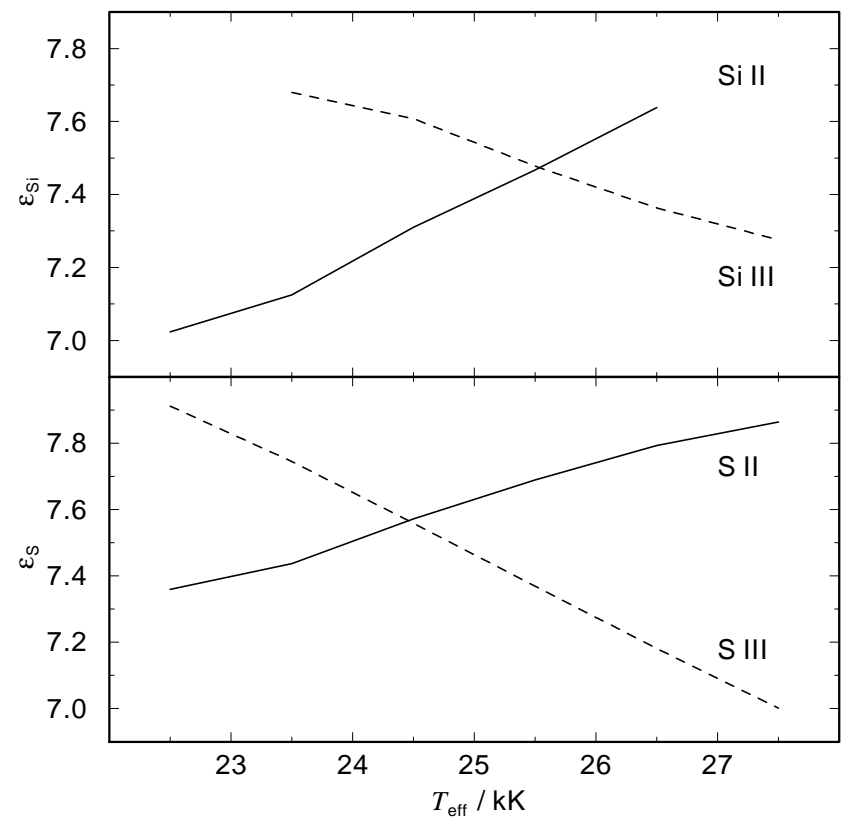

Figure 2. Abundance $\varepsilon_{i}$ measured based on Si II and Si III lines (top panel) and on S II and SiII lines (bottom) as a function of temperature for $\log g=5.3$.

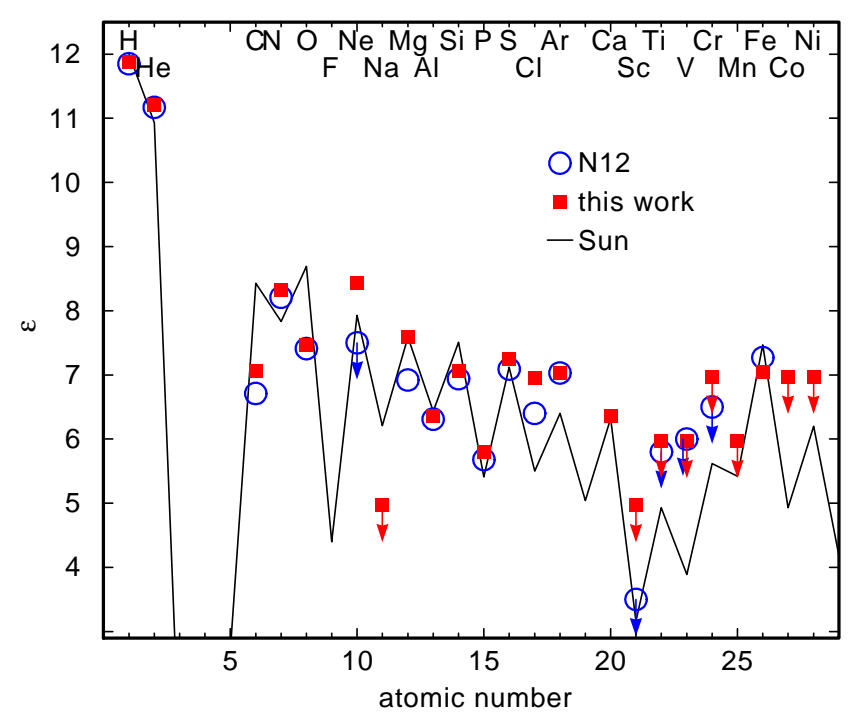

Figure 3. Photospheric abundances $\epsilon_{i}$ of CPD-20 21123 determined from line profile fits compared to the results from N12 and solar values from Asplund et al. (2009); Scott et al. (2015a,b); Grevesse et al. (2015). Upper limits are indicated with arrows.

grid used.

Figure 8 shows the departure coefficients of the levels of $\mathrm{H}$. While the departure coefficient of the lowest level is below unity in the deepest part of the line-forming region, it reaches up to three in the outer part. This coefficient determines the relation between the local temperatures of a NLTE and LTE model. If it is below unity, it requires $T_{\mathrm{NLTE}}<T_{\mathrm{LTE}}$ (Kudritzki 1979). In the formation depth of the Balmer lines, the departure coefficients of the $\mathrm{H}$ levels deviate from each
Table 1. Abundances $\epsilon_{i}$ for analyzed lines of $\mathrm{Cl}$ II and Fe III.

\begin{tabular}{cccc}
\hline \hline & \multicolumn{3}{c}{ Abundance } \\
Ion & $\lambda / \AA$ & This work & $\mathrm{N} 12$ \\
\hline \multirow{2}{*}{ Cl II } & 4794.6 & 7.24 & 6.38 \\
& 4810.1 & 7.36 & 6.43 \\
& 4896.8 & 6.94 & 6.96 \\
Fe III & 3954.3 & 7.34 & \\
& 4053.1 & 6.83 & \\
& 4081.0 & 7.07 & \\
& 4122.0 & 6.80 & \\
& 4137.8 & 7.12 & \\
& 4139.4 & 6.80 & \\
& 4164.7 & 6.97 & 7.13 \\
& 4166.8 & 7.17 & 7.38 \\
& 4238.6 & 7.34 & \\
& 4304.8 & 7.64 & \\
& 4310.4 & 7.81 & \\
& 4395.8 & 7.17 & 7.16 \\
& 4419.6 & 7.61 & 7.30 \\
& 4431.0 & 7.73 & \\
\hline
\end{tabular}

Notes. Abundances in the form $\epsilon_{i}=\log \left(n_{i} / n_{\mathrm{H}}\right)+12$, with the number densities $n_{i}$ and $n_{\mathrm{H}}$ of elements $i$ and $\mathrm{H}$.

other (Fig. 8). This might be a reason for discrepancies in the Balmer-line cores that were reported in LTE analyses (Napiwotzki 1997).

As described in Sect. 4 and shown in Fig. 2, we see a discrepancy in the temperature determined from Si II/III and S II/III that was also reported by N12. To evaluate the importance of NLTE effects for the lines of Si and S and thus on the temperature determination, we investigated also the departure coefficients for the levels corresponding to the lines used for the abundance determinations (Fig. 2). The departure coefficients are below unity in the line-forming region (Fig. 6), However, when comparing the TMAD LTE and NLTE models, the line profiles coincide within the thickness of a line. This result validates the well established practice to use LTE codes for the analysis of sdB stars.

\subsection{Mass, spectral type, and distance}

We determine the mass of CPD $-20^{\circ} 1123$ by comparing its position in the $T_{\text {eff }}-\log g$ diagram with evolutionary tracks for sdB stars from Dorman et al. (1993, $\left.Z=Z_{\odot}, Y=Y_{\odot}\right)$ (Fig. 9) and find $M=0.475 \pm 0.015 M_{\odot}$ which is close to the assumed typical sdB mass of $M=0.47 M_{\odot}$ from (N12). The error includes the propagation of uncertainties in $T_{\text {eff }}$ and $\log g$ as well as in the abundances, that we estimated from tracks for different compositions. However, we want to note that this mass determination has to be treated with caution, since, as discussed in Section 1, the intermediate He abundance might indicate a pre-EHB nature of the object. Different pre-EHB evolutionary tracks for the hot-flasher scenario exist (e.g., Cassisi et al. 2003; Miller Bertolami et al. 2008; Battich et al. 2018) but the mass determination from these is difficult since they differ depending on the evolutionary phase when the flash occurs, i.e., whether it is a early or late hot flasher with deep or shallow mixing. Furthermore, these tracks might be inappropriate as well due to the fact that CPD-20 1123 is in a short period binary with a low-mass 


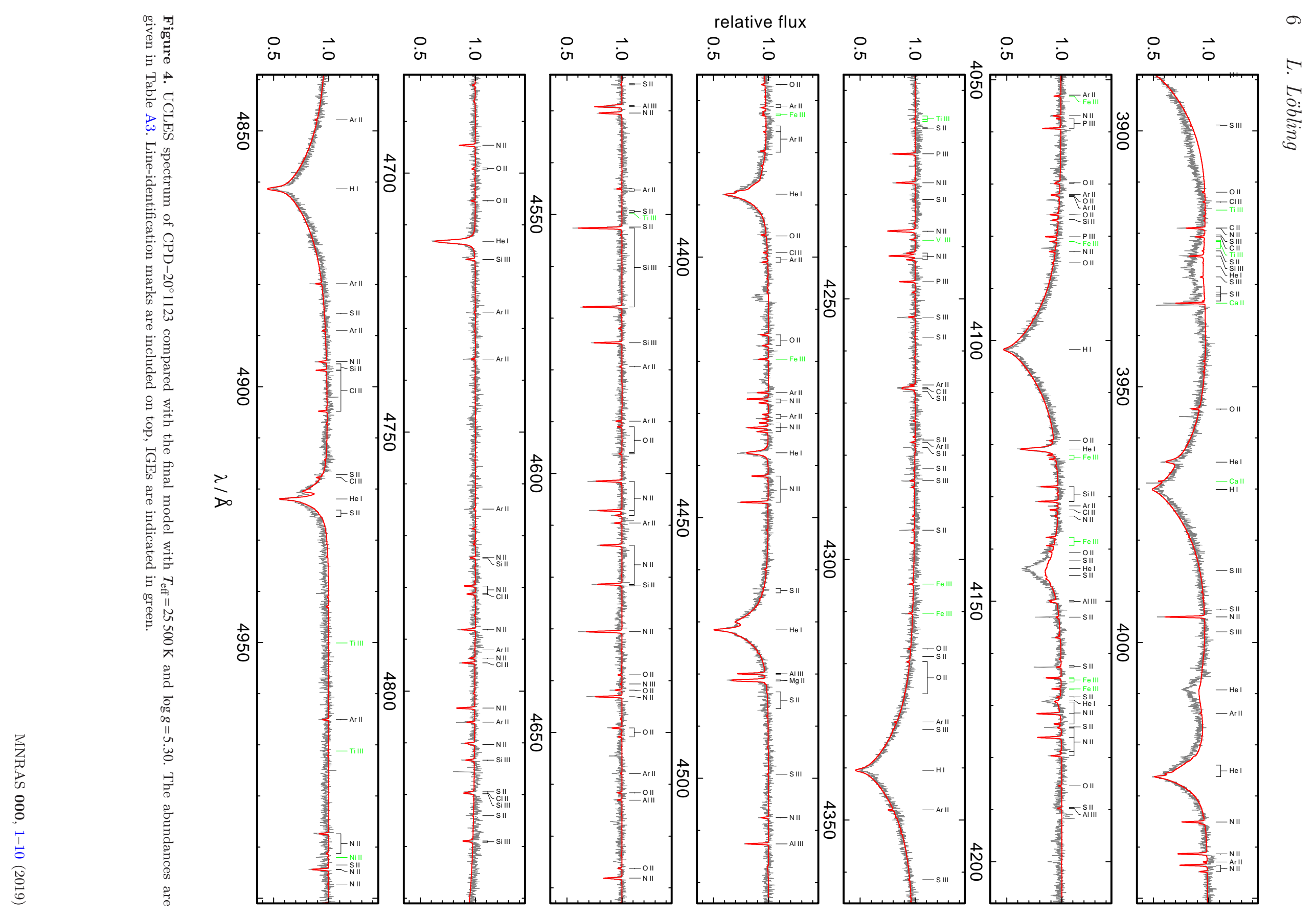




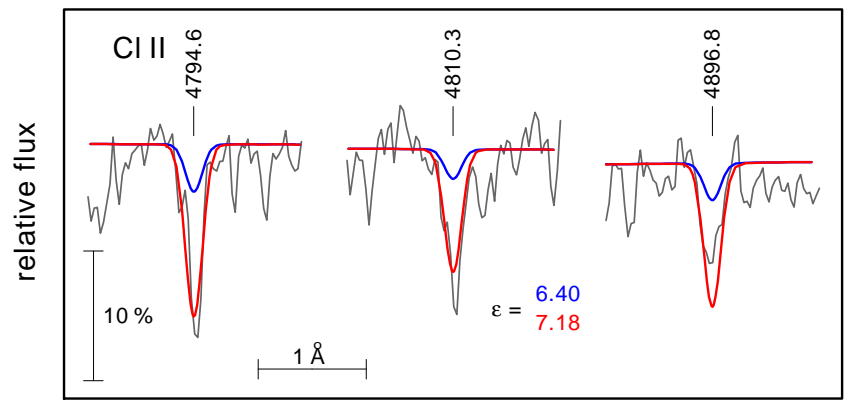

$\Delta \lambda / \AA$

Figure 5. Synthetic spectra calculated with $\log g=5.30, T_{\text {eff }}=$ 25500 , and $\mathrm{Cl}$ abundances of $\log \varepsilon=6.40$ (blue) and 7.18 (red), compared with the UCLES observations of lines of $\mathrm{Cl}$ II.

main-sequence companion or a white dwarf (WD, N12), which suggests that the sdB star formed via a binary channel and lost its envelope in a mass-transfer event rather than in a hot-flasher scenario. From the position in the color-color diagram and a spectral energy distribution (SED, Fig. A2) similar to G191-B2B, Caballero \& Solano (2007) classified CPD-20 1123 as an early DA-type WD. Both stars' 2MASS $K_{S}$ magnitudes are identical but G191-B2B is brighter in the B-band. Assuming the same spectral type, they concluded, that $\mathrm{CPD}-20^{\circ} 1123$ should be colder and, thus, closer to the Sun compared to G191-B2B. We find, that our best model for $\mathrm{CPD}-20^{\circ} 1123$ resembles the observed magnitudes better than a model SED for G191-B2B $\left(T_{\text {eff }}=60000 \mathrm{~K}\right.$, $\log g=7.6$, obtained from TheoSSA${ }^{4}$, Fig. A2, Rauch et al. 2013). Including also the GALEX FUV and NUV magnitude (Bianchi et al. 2017), we can furthermore confirm, that the observations are affected by interstellar reddening of $E_{\mathrm{B}-\mathrm{V}}=0.0684$ (Schlafly \& Finkbeiner 2011). With the knowledge of $\log g$ from spectral analyses, Vennes et al. (2007) revised the spectral type and classified CPD-20 1123 as an sdB. Following the same arguments as above, we can now conclude from the identical $K_{S}$ magnitude, that CPD-201123 should be further away from the Sun compared to G191-B2B. This is confirmed by the distances from Gaia parallaxes (Bailer-Jones et al. 2018), namely $329 \pm 7$ pc for $\mathrm{CPD}-20^{\circ} 1123$ and $52.85 \pm 0.19$ for G191-B2B. The precise Gaia distance is an excellent test for the atmospheric parameters. With the values from Table A3, we get a spectroscopic distance of $425_{-177}^{+128} \mathrm{pc}$ which is still in agreement with the Gaia value. The surface gravity determined in previous studies is too low since $\log g=4.9 \pm 0.1$ (N12) would yield a distance of $678_{-102}^{+87}$ pc.

Using the Gaia distance, and our atmospheric parameters, we can test the mass determination from evolutionary tracks. We find $M=0.24_{-0.12}^{+0.24}$ which is too low for a typical sdB star but just in agreement with the other method. To increase this value to 0.475 , a higher surface gravity of $\log g=5.6$ would be needed, and is within the observational error.

\footnotetext{
${ }^{4}$ http://dc.g-vo.org/theossa
}

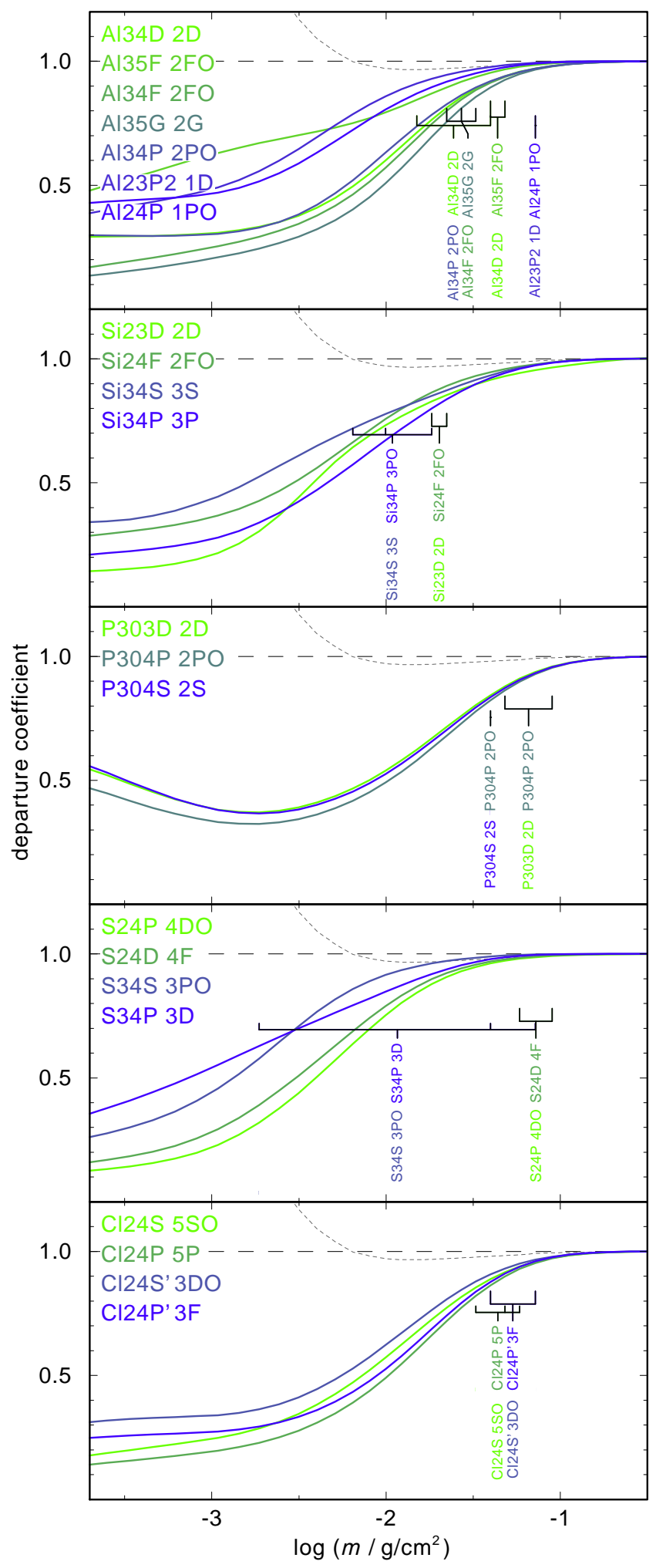

Figure 6. Departure coefficients of the $\mathrm{Al}$ II, AlıII, Si II, SiIII, P III, S II, S III, and Cl II levels corresponding to the lines in Figs. 2, $\mathrm{A} 3$, and 5. The formation depth of the line cores is indicated. The departure coefficient of the lowest $\mathrm{H}$ level is included (gray, dashed). 


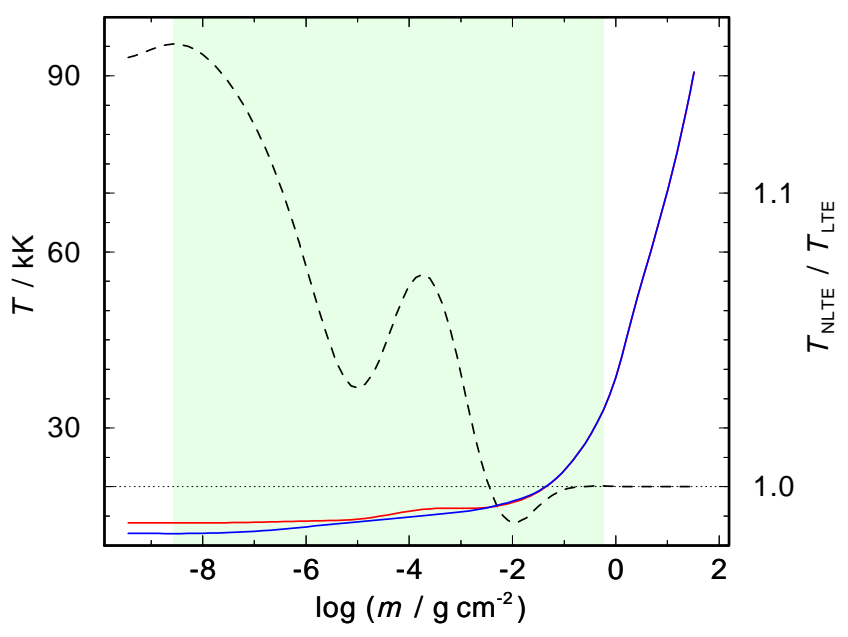

Figure 7. Temperature structure of a NLTE (red) and a LTE model (blue). Both are calculated with $T_{\text {eff }}=24500 \mathrm{~K}$ and $\log g=4.9 . T_{\mathrm{NLTE}} / T_{\mathrm{LTE}}$ is indicated as dashed line. The line-forming region is indicated in green.

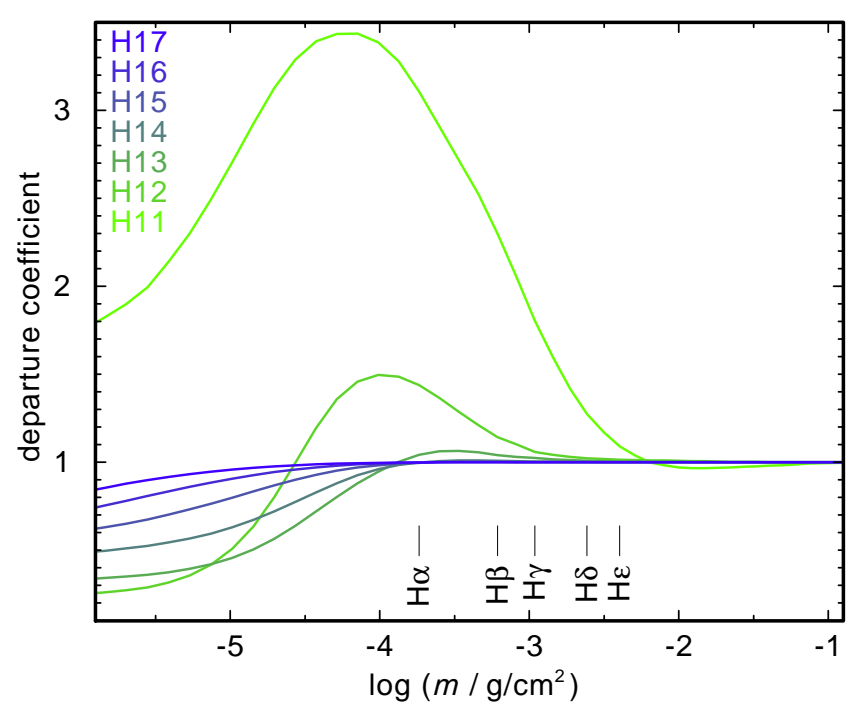

Figure 8. Departure coefficients of the $\mathrm{H}$ levels and formation depth of the cores of the Balmer lines.

\section{CONCLUSIONS}

In the course of this work, we extended our model-atom database TMAD by constructing new model atoms for $\mathrm{Al}$, $\mathrm{P}$, and $\mathrm{Cl}$. The low ionization stages (II-IV) of F, S, and Ar were substantially enlarged. The analysis of the optical spectrum of the intermediate He-rich sdB star CPD-20 1123 constitutes a test of NLTE stellar atmosphere code TMAP for the low- $T_{\text {eff }}$ regime. This star is located close the hightemperature edge until which it may be justified to use LTE model atmospheres for the spectral analysis. However, NLTE effects are present in every star and become visible, at least, in the analysis of high-resolution spectra and in high-energy observations. We found that the departure coefficients differ from unity in the line-forming region. This effect is however not strong enough to cause visible differ-

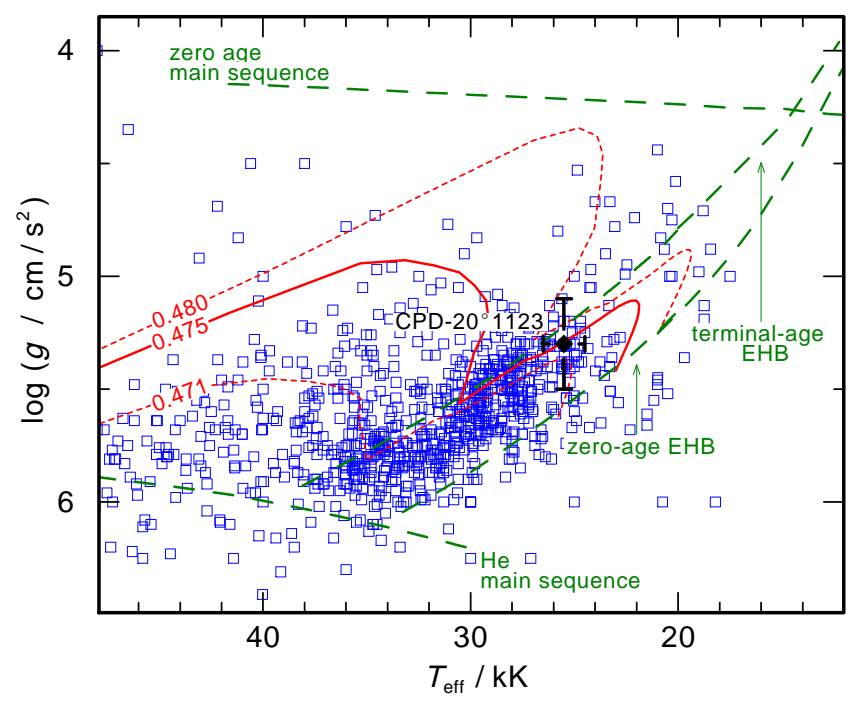

Figure 9. Location of CPD-20 1123 (black, with error bars) in the $T_{\text {eff }}-\log g$ diagram compared with sd(O)B-type stars close to the extended horizontal branch (EHB) from Geier et al. (2017, blue squares). Post-EHB evolutionary tracks from Dorman et al. (1993, red, $\left.Z=Z_{\odot}, Y=Y_{\odot}\right)$ are included and labeled with stellar mass.

ences in the line profiles comparing NLTE and LTE atmospheres. We determine $T_{\text {eff }}=25500 \pm 1000 \mathrm{~K}$ which is $2000 \mathrm{~K}$ higher than the value found by N12 in their LTE analysis. The surface gravity of $\log g=5.3 \pm 0.3$ is also higher by 0.4 dex which is favored by the Gaia distance measurement. Our abundances for the light metals are higher up to a factor of 7 compared to those of N12. Fe is the only element, for which we determine a slightly lower abundance. Our comparison of a NLTE and LTE model calculated with TMAP for the same atmospheric parameters and abundances ruled out that these discrepancies are NLTE effects, since the synthetic spectra for these two models differ only marginally. This work proves that with the presented extension of the atomic database for low ionization stages, NLTE and LTE codes are equally well suited for the analysis of sdB stars.

\section{ACKNOWLEDGEMENTS}

The author thanks Thomas Rauch for the idea and initiation of this project and the helpful discussions and comments and Klaus Werner for the help with the draft of this publication. I thank Michael Knörzer and Stefanie Klein for the preparation of some of the new TMAD model atoms. The author thanks the anonymous referee for the very constructive comments and corrections that improved the quality of this work. I thank Simon Jeffery for the discussions about the object and this work and for the very helpful comments and corrections during the review process. We thank Naslim Neelamkodan and Simon Jeffery for putting the reduced UCLES spectra at our disposal. We thank John Pritchard from the ESO User Support Department for the explanations about FEROS and the data reduction process. This work was supported by the German Research Foundation (DFG, grant WE 1312/49- 
1). We were supported by the High Performance and Cloud Computing Group at the Zentrum für Datenverarbeitung of the University of Tübingen, the state of Baden-Württemberg through bwHPC, and the DFG (grant INST 37/935-1 FUGG). The GAVO project had been supported by the Federal Ministry of Education and Research (BMBF) at Tübingen (05 AC 6 VTB, 05 AC 11 VTB). The TIRO (http://astro-uni-tuebingen.de/ ${ }^{\sim T I R O), ~ T M A D ~}$ (http://astro-uni-tuebingen.de/ TMAD), and TheoSSA (http://dc.g-vo.org/theossa) services used for this paper were constructed as part of the activities of the German Astrophysical Virtual Observatory. This research has made use of NASA's Astrophysics Data System and the SIMBAD database, operated at CDS, Strasbourg, France. This work has made use of data from the European Space Agency (ESA) mission Gaia (https://www.cosmos.esa.int/gaia), processed by the Gaia Data Processing and Analysis Consortium (DPAC, https://www.cosmos.esa.int/web/gaia/ dpac/consortium). Funding for the DPAC has been provided by national institutions, in particular the institutions participating in the Gaia Multilateral Agreement.

\section{REFERENCES}

Abou El-Maaref A., Uosif M. A. M., Allam S. H., El-Sherbini T. M., 2012, Atomic Data and Nuclear Data Tables, 98, 589 Ahmad A., Jeffery C. S., 2006, Balt. Astron., 15, 139

Asplund M., Grevesse N., Sauval A. J., Scott P., 2009, ARA\&A, 47,481

Bailer-Jones C. A. L., Rybizki J., Fouesneau M., Mantelet G., Andrae R., 2018, AJ, 156, 58

Barnard A. J., Cooper J., Shamey L. J., 1969, A\&A, 1, 28

Barnard A. J., Cooper J., Smith E. W., 1974, J. Quant. Spectrosc. Radiative Transfer, 14, 1025

Battich T., Miller Bertolami M. M., Córsico A. H., Althaus L. G., 2018, A\&A, 614, A136

Beauchamp A., Wesemael F., Bergeron P., 1997, ApJS, 108, 559

Becker S. R., Butler K., 1989, A\&A, 209, 244

Bengtson R. D., 1968, PhD thesis, Inst. for Fluid Dynamics a. Appl. Math., Univ. of Maryland

Bianchi L., Shiao B., Thilker D., 2017, ApJS, 230, 24

Caballero J. A., Solano E., 2007, ApJ, 665, L151

Cassisi S., Schlattl H., Salaris M., Weiss A., 2003, ApJ, 582, L43

Cowley C. R., 1970, The theory of stellar spectra. Gordon \& Breach, New York

Cowley C. R., 1971, The Observatory, 91, 139

Cutri R. M., et al., 2003, VizieR Online Data Catalog, p. II/246

Dimitrijević M. S., Sahal-Bréchot S., 1990, A\&AS, 82, 519

Dimitrijević M. S., Sahal-Bréchot S., 1993, A\&AS, 99, 585

Dorman B., Rood R. T., O'Connell R. W., 1993, ApJ, 419, 596

Eriksson K. B. S., 1983, Phys. Scr., 28, 593

Foster E. W., 1962, Proceedings of the Physical Society, 80, 882

Fuhr J. R., Wiese W. L., 1998, CRC Handbook of Chemistry and Physics. CRC Press

Geier S., Østensen R. H., Nemeth P., Gentile Fusillo N. P., Gänsicke B. T., Telting J. H., Green E. M., Schaffenroth J., 2017, A\&A, 600, A50

Gill D., Kapteyn J. C., 1896, Annals of the Cape Observatory, 3,1

Green R. F., Schmidt M., Liebert J., 1986, ApJS, 61, 305

Grevesse N., Scott P., Asplund M., Sauval A. J., 2015, A\&A, 573, A27

Griem H. R., 1974, Spectral line broadening by plasmas

Heber U., 2016, PASP, 128, 082001

Hubeny I., Hummer D. G., Lanz T., 1994, A\&A, 282, 151
Hummer D. G., Mihalas D., 1988, ApJ, 331, 794

Kudritzki R. P., 1979, in Boury A., Grevesse N., Remy-Battiau L., eds, Vol. 22, Liege International Astrophysical Colloquia. pp 295-318

Kupfer T., et al., 2015, A\&A, 576, A44

Kurucz R. L., 1991, in Crivellari L., Hubeny I., Hummer D. G., eds, NATO ASIC Proc. 341: Stellar Atmospheres - Beyond Classical Models. p. 441

Kurucz R. L., 2009, in Hubeny I., Stone J. M., MacGregor K., Werner K., eds, American Institute of Physics Conference Series Vol. 1171, American Institute of Physics Conference Series. p. 43, doi:10.1063/1.3250087

Lemke M., 1997, A\&AS, 122, 285

Miller Bertolami M. M., Althaus L. G., Unglaub K., Weiss A., 2008, A\&A, 491, 253

Napiwotzki R., 1997, A\&A, 322, 256

Naslim N., Jeffery C. S., Ahmad A., Behara N. T., Şahìn T., 2010, MNRAS, 409, 582

Naslim N., Geier S., Jeffery C. S., Behara N. T., Woolf V. M., Classen L., 2012, MNRAS, 423, 3031

Rauch T., Deetjen J. L., 2003, in Hubeny I., Mihalas D., Werner K., eds, Astronomical Society of the Pacific Conference Series Vol. 288, Stellar Atmosphere Modeling. p. 103 (arXiv: astro-ph/0403239)

Rauch T., Werner K., Bohlin R., Kruk J. W., 2013, A\&A, 560, A106

Rauch T., Gamrath S., Quinet P., Löbling L., Hoyer D., Werner K., Kruk J. W., Demleitner M., 2017, A\&A, 599, A142

Savitzky A., Golay M. J. E., 1964, Analytical Chemistry, 36, 1627

Schindewolf M., Németh P., Heber U., Battich T., Miller Bertolami M. M., Irrgang A., Latour M., 2018, A\&A, 620, A36

Schlafly E. F., Finkbeiner D. P., 2011, ApJ, 737, 103

Scott P., et al., 2015a, A\&A, 573, A25

Scott P., Asplund M., Grevesse N., Bergemann M., Sauval A. J., 2015b, A\&A, 573, A26

Seaton M. J., 1987, Journal of Physics B Atomic Molecular Physics, 20, 6363

Tremblay P.-E., Bergeron P., 2009, ApJ, 696, 1755

Varsavsky C. M., 1961, ApJS, 6, 75

Vennes S., Kawka A., Smith J. A., 2007, ApJ, 668, L59

Werner K., Deetjen J. L., Dreizler S., Nagel T., Rauch T., Schuh S. L., 2003, in Hubeny I., Mihalas D., Werner K., eds, Astronomical Society of the Pacific Conference Series Vol. 288, Stellar Atmosphere Modeling. p. 31 (arXiv:astro-ph/0209535)

Werner K., Dreizler S., Rauch T., 2012, TMAP: Tübingen NLTE Model-Atmosphere Package, Astrophysics Source Code Library [record ascl:1212.015] (ascl:1212.015)

Wiese W. L., Smith M. W., Miles B. M., 1969, Atomic transition probabilities. Vol. 2: Sodium through Calcium. A critical data compilation

Zacharias N., Finch C. T., Girard T. M., Henden A., Bartlett J. L., Monet D. G., Zacharias M. I., 2012, VizieR Online Data Catalog, p. I/322A 
10 L. Löbling

APPENDIX A: ADDITIONAL FIGURES AND

TABLES.

This paper has been typeset from a $\mathrm{T}_{\mathrm{E}} \mathrm{X} / \mathrm{LAT} \mathrm{E} \mathrm{X}$ file prepared by the author. 
Table A1. Statistics of the $\mathrm{H}-\mathrm{Ar}^{a}$ and $\mathrm{Ca}-\mathrm{Ni}^{b}$ model atoms used in our model-atmosphere calculations.

\begin{tabular}{|c|c|c|c|c|c|c|c|c|c|}
\hline \multirow{2}{*}{\multicolumn{2}{|c|}{ Ion }} & \multicolumn{2}{|c|}{ Levels } & \multirow{2}{*}{ Lines } & \multirow{2}{*}{\multicolumn{2}{|c|}{ Ion }} & \multirow{2}{*}{$\begin{array}{l}\text { Super } \\
\text { levels }\end{array}$} & \multirow{2}{*}{$\begin{array}{l}\text { Super } \\
\text { lines }\end{array}$} & \multirow{2}{*}{$\begin{array}{c}\text { Individual } \\
\text { lines }\end{array}$} \\
\hline & & NLTE & $\overline{\mathrm{LTE}}$ & & & & & & \\
\hline \multirow{2}{*}{$\mathrm{H}$} & I & 10 & 22 & 45 & $\mathrm{Ca}$ & II & 7 & 26 & 2612 \\
\hline & II & 1 & - & - & & III & 7 & 26 & 40664 \\
\hline \multirow[t]{3}{*}{$\mathrm{He}$} & I & 103 & 0 & 504 & & IV & 1 & 0 & 0 \\
\hline & II & 16 & 16 & 120 & $\mathrm{Sc}$ & II & 7 & 26 & 77014 \\
\hline & III & 1 & - & - & & III & 7 & 26 & 1299 \\
\hline \multirow[t]{3}{*}{$\mathrm{C}$} & II & 10 & 36 & 14 & & IV & 1 & 0 & 0 \\
\hline & III & 16 & 89 & 30 & $\mathrm{Ti}$ & II & 7 & 27 & 312054 \\
\hline & IV & 1 & 0 & 0 & & III & 7 & 25 & 46707 \\
\hline \multirow[t]{4}{*}{$\mathrm{N}$} & I & 10 & 17 & 11 & & IV & 7 & 24 & 2226 \\
\hline & II & 16 & 232 & 24 & & $\mathrm{~V}$ & 1 & 0 & 0 \\
\hline & III & 16 & 50 & 34 & $\mathrm{Cr}$ & II & 7 & 27 & 728080 \\
\hline & IV & 1 & 0 & 0 & & III & 7 & 27 & 1421382 \\
\hline \multirow[t]{3}{*}{$\mathrm{O}$} & I & 10 & 86 & 7 & & IV & 7 & 23 & 234170 \\
\hline & II & 16 & 31 & 26 & & $\mathrm{~V}$ & 1 & 0 & 0 \\
\hline & III & 1 & 0 & 0 & Mn & II & 7 & 27 & 136814 \\
\hline \multirow{2}{*}{$\mathrm{F}$} & II & 10 & 98 & 8 & & III & 7 & 27 & 1668146 \\
\hline & III & 1 & 0 & 0 & & IV & 7 & 25 & 719387 \\
\hline \multirow{3}{*}{$\mathrm{Ne}$} & I & 6 & 32 & 3 & & $\mathrm{~V}$ & 1 & 0 & 0 \\
\hline & II & 15 & 19 & 21 & $\mathrm{Fe}$ & II & 7 & 27 & 531460 \\
\hline & III & 1 & 0 & 0 & & III & 7 & 25 & 537689 \\
\hline \multirow[t]{2}{*}{$\mathrm{Mg}$} & II & 14 & 16 & 34 & & IV & 7 & 25 & 3102371 \\
\hline & III & 1 & 0 & 0 & & $\mathrm{~V}$ & 1 & 0 & 0 \\
\hline \multirow[t]{3}{*}{$\mathrm{Al}$} & II & 10 & 118 & 12 & Co & II & 7 & 27 & 593140 \\
\hline & III & 12 & 28 & 25 & & III & 7 & 27 & 1325205 \\
\hline & IV & 1 & 0 & 0 & & IV & 7 & 27 & 552916 \\
\hline \multirow[t]{3}{*}{$\mathrm{Si}$} & II & 13 & 45 & 28 & & $\mathrm{~V}$ & 1 & 0 & 0 \\
\hline & III & 16 & 94 & 25 & $\mathrm{Ni}$ & II & 7 & 27 & 322269 \\
\hline & IV & 1 & 0 & 0 & & III & 7 & 22 & 1033920 \\
\hline \multirow[t]{3}{*}{$\mathrm{P}$} & II & 8 & 38 & 2 & & IV & 7 & 25 & 2512561 \\
\hline & III & 15 & 39 & 4 & & $\mathrm{~V}$ & 1 & 0 & 0 \\
\hline & IV & 1 & 0 & 0 & & & & & \\
\hline \multirow[t]{3}{*}{$\mathrm{S}$} & II & 16 & 220 & 18 & & & & & \\
\hline & III & 15 & 216 & 12 & & & & & \\
\hline & IV & 1 & 0 & 0 & & & & & \\
\hline \multirow[t]{3}{*}{$\mathrm{Cl}$} & II & 11 & 75 & 5 & & & & & \\
\hline & III & 16 & 18 & 15 & & & & & \\
\hline & IV & 1 & 0 & 0 & & & & & \\
\hline \multirow[t]{3}{*}{$\mathrm{Ar}$} & II & 10 & 238 & 4 & & & & & \\
\hline & III & 15 & 268 & 6 & & & & & \\
\hline & IV & 1 & 0 & 0 & & & & & \\
\hline total & & 446 & 2141 & 1037 & & & 155 & 568 & 15902086 \\
\hline
\end{tabular}

Notes. ${ }^{(a)}$ classical model atoms, ${ }^{(b)}$ model atoms constructed using a statistical approach (Rauch \& Deetjen 2003), (c) treated as NLTE levels. 


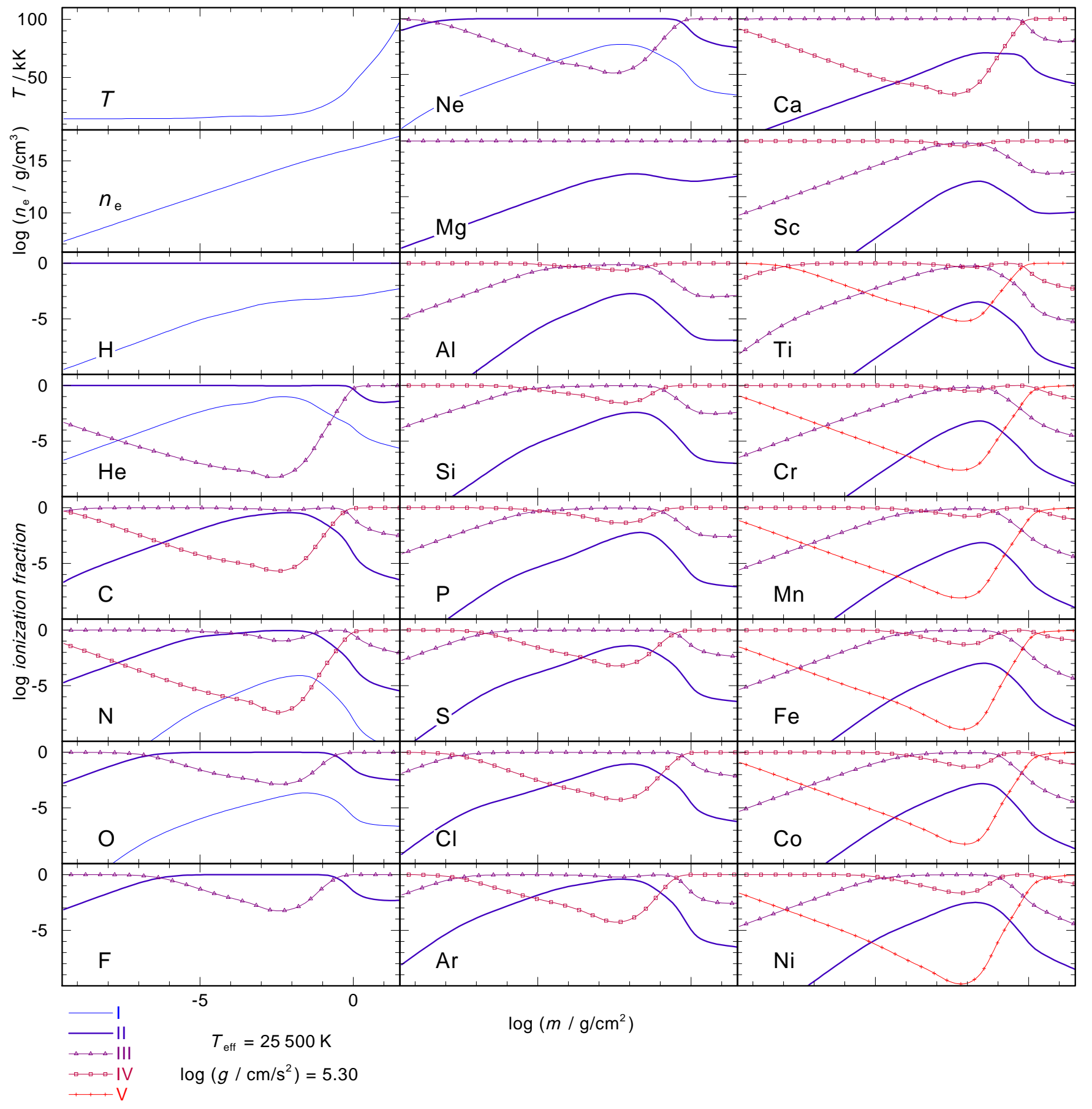

Figure A1. Temperature and electron density structure and ionization fractions of all ions which are considered in our final model for CPD $-20^{\circ} 1123$. 


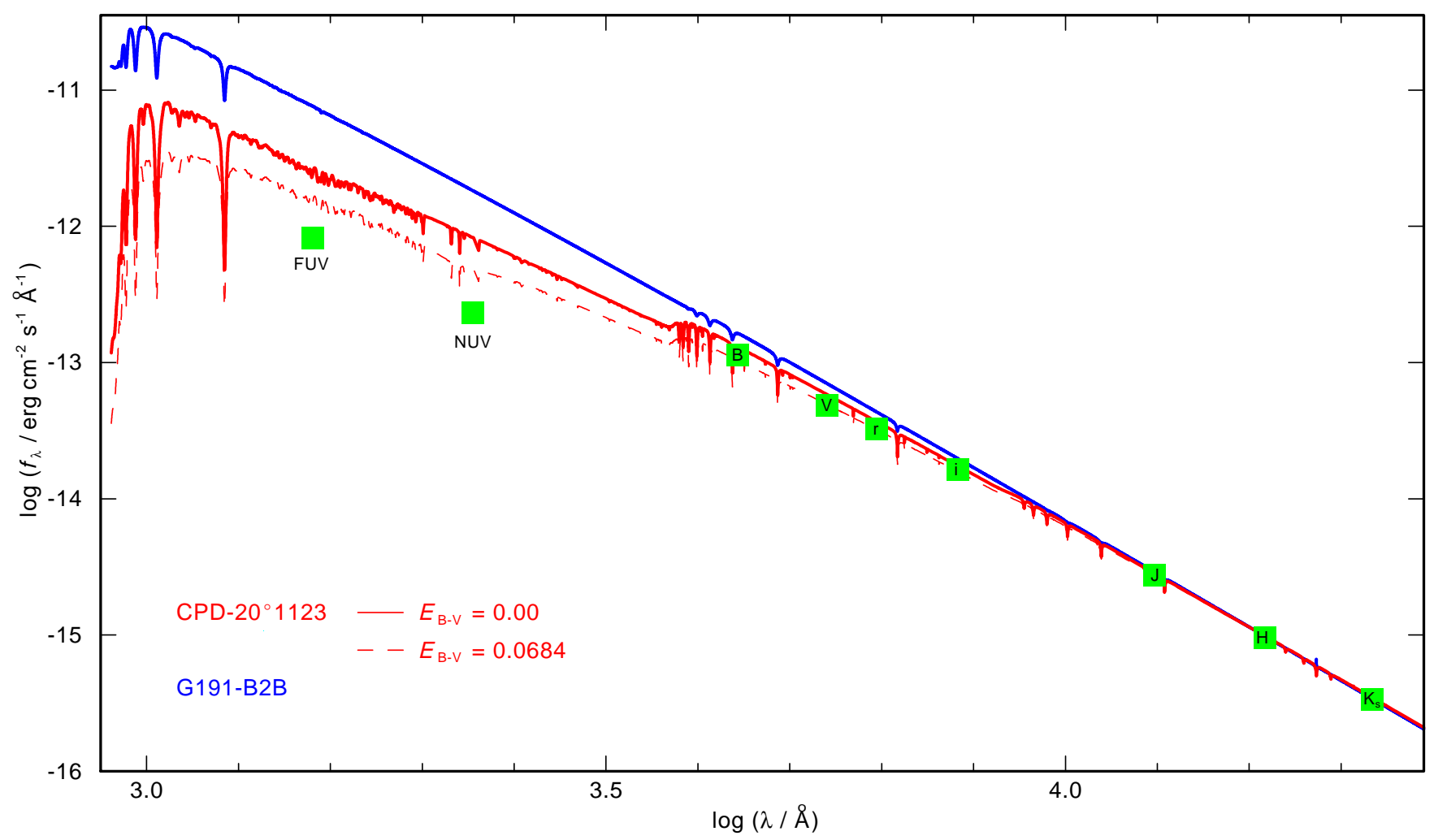

Figure A2. Synthetic spectra of our best model of CPD $-20^{\circ} 1123$ without reddening (thick, red line) and with interstellar reddening with $E_{\mathrm{B}-\mathrm{V}}=0.0684$ (dashed, red) compared with the SED of the DA WD G191-B2B (blue). The model fluxes are normalized to the 2MASS $K_{S}$ magnitude $(\lambda=21590 \AA$, Cutri et al. 2003). $B, r$, and $i$ magnitudes from Zacharias et al. (2012), the $V$ magnitude from Kupfer et al. (2015), and the GALEX FUV and NUV magnitudes from Bianchi et al. (2017) were added.

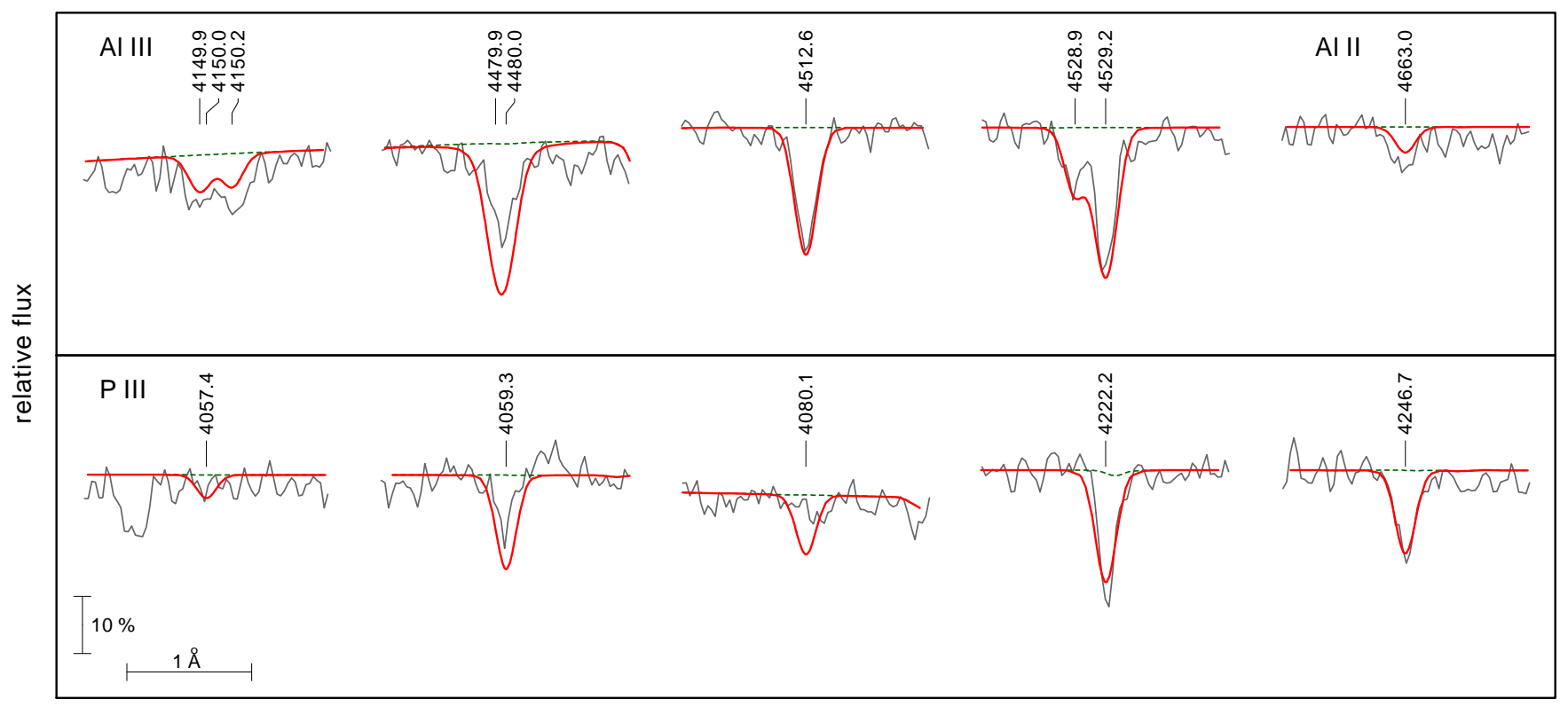

$\Delta \lambda / \AA$

Figure A3. Synthetic spectra calculated with $\log g=5.30, T_{\mathrm{eff}}=25500$, and final abundances from Table A3, compared with the UCLES observations of lines of $\mathrm{Al}$ II, $\mathrm{Al}$ III, and P III. 


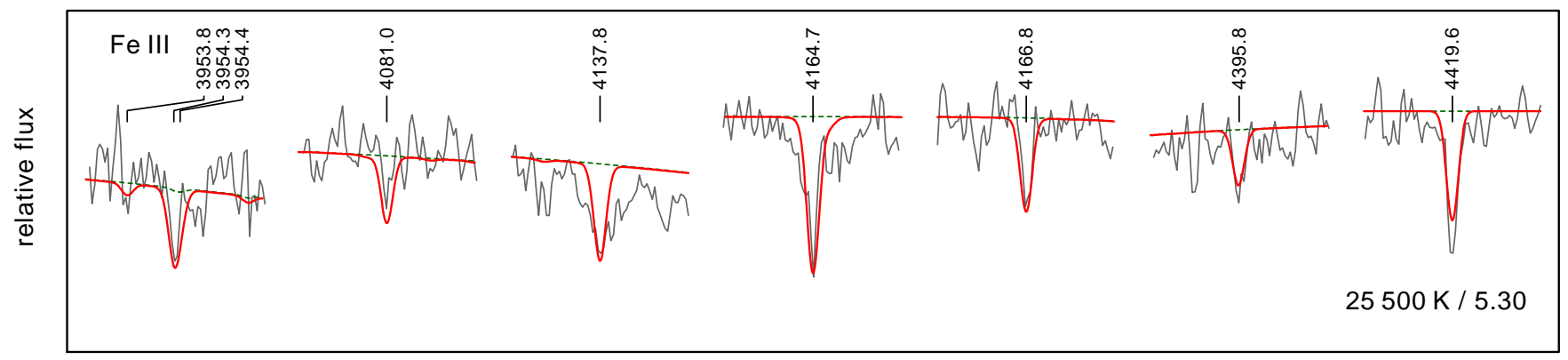

Figure A4. Synthetic spectra calculated with $\log g=5.30, T_{\text {eff }}=25500$, and final abundances from Table A3, compared with the UCLES observations of lines of Fe III. 
Table A2. $\mathrm{H}$ and He lines used for the determination of atmospheric parameters.

\begin{tabular}{|c|c|}
\hline Ion & $\lambda / \AA$ \\
\hline \multicolumn{2}{|c|}{ UCLES and FEROS range } \\
\hline H I & 3970.08 \\
\hline He I & 4026.98 \\
\hline H I & 4101.71 \\
\hline H I & 4340.46 \\
\hline He I & 4387.93 \\
\hline He I & 4437.55 \\
\hline He I & 4471.50 \\
\hline $\mathrm{H} \mathrm{I}$ & 4861.32 \\
\hline He I & 4921.93 \\
\hline \multicolumn{2}{|c|}{ only FEROS range } \\
\hline He I & 5015.68 \\
\hline He I & 5047.74 \\
\hline He I & 5875.75 \\
\hline H I & 6562.79 \\
\hline He I & 6678.15 \\
\hline
\end{tabular}


Table A3. Parameters of CPD-20²1123.

\begin{tabular}{|c|c|c|c|c|c|}
\hline$T_{\text {eff }} / \mathrm{K}$ & \multicolumn{5}{|c|}{$25500 \pm 1000$} \\
\hline $\log \left(g / \mathrm{cm} / \mathrm{s}^{2}\right)$ & \multicolumn{5}{|c|}{$5.30 \pm 0.3$} \\
\hline$d / \mathrm{pc}$ & \multicolumn{5}{|c|}{$329 \pm 7^{(a)}$} \\
\hline$M / M_{\odot}$ & \multicolumn{5}{|c|}{$0.475 \pm 0.015^{(b)}$} \\
\hline$E_{\mathrm{B}-\mathrm{V}}$ & \multicolumn{5}{|c|}{$0.0684 \pm 0.0009^{(c)}$} \\
\hline GALEX FUV & \multicolumn{5}{|c|}{$11.9094 \pm 0.0045^{(d)}$} \\
\hline GALEX NUV & \multicolumn{5}{|c|}{$12.4470 \pm 0.0038^{(d)}$} \\
\hline$B$ & \multicolumn{5}{|c|}{$11.907 \pm 0.06^{(e)}$} \\
\hline$V$ & \multicolumn{5}{|c|}{$12.173 \pm 0.12^{(f)}$} \\
\hline$r$ & \multicolumn{5}{|c|}{$12.336 \pm 0.01^{(e)}$} \\
\hline$i$ & \multicolumn{5}{|c|}{$12.638 \pm 0.06^{(e)}$} \\
\hline$J$ & \multicolumn{5}{|c|}{$12.56 \pm 0.02^{(g)}$} \\
\hline$H$ & \multicolumn{5}{|c|}{$12.66 \pm 0.03^{(g)}$} \\
\hline$K_{S}$ & \multicolumn{5}{|c|}{$12.76 \pm 0.03^{(g)}$} \\
\hline Abundances & {$[\mathrm{X}]$} & Mass fraction & Number fraction & $\varepsilon$ & {$[\mathrm{X} / \mathrm{Fe}]$} \\
\hline $\mathrm{H}$ & -0.06 & $6.37 \times 10^{-1}$ & $8.77 \times 10^{-1}$ & 11.95 & 0.21 \\
\hline $\mathrm{He}$ & 0.15 & $3.52 \times 10^{-1}$ & $1.22 \times 10^{-1}$ & 11.09 & 0.42 \\
\hline $\mathrm{C}$ & -0.88 & $3.12 \times 10^{-4}$ & $3.61 \times 10^{-5}$ & 7.57 & -0.61 \\
\hline $\mathrm{N}$ & 0.71 & $3.52 \times 10^{-3}$ & $3.49 \times 10^{-4}$ & 8.55 & 0.98 \\
\hline $\mathrm{O}$ & -1.16 & $3.94 \times 10^{-4}$ & $3.42 \times 10^{-5}$ & 7.54 & -0.89 \\
\hline $\mathrm{Ne}$ & 0.23 & $2.14 \times 10^{-3}$ & $1.47 \times 10^{-4}$ & 8.18 & 0.50 \\
\hline $\mathrm{Na}$ & $\leq-1.26$ & $\leq 1.50 \times 10^{-6}$ & $\leq 1.00 \times 10^{-7}$ & $\leq 4.97$ & $\leq-0.98$ \\
\hline $\mathrm{Mg}$ & 0.18 & $1.04 \times 10^{-3}$ & $5.93 \times 10^{-5}$ & 7.78 & 0.44 \\
\hline $\mathrm{Al}$ & 0.47 & $1.55 \times 10^{-4}$ & $7.99 \times 10^{-6}$ & 6.91 & 0.72 \\
\hline $\mathrm{Si}$ & -0.05 & $5.87 \times 10^{-4}$ & $2.90 \times 10^{-5}$ & 7.47 & 0.22 \\
\hline $\mathrm{P}$ & 0.80 & $3.64 \times 10^{-5}$ & $1.63 \times 10^{-6}$ & 6.22 & 1.07 \\
\hline $\mathrm{S}$ & 0.43 & $8.34 \times 10^{-4}$ & $3.61 \times 10^{-5}$ & 7.57 & 0.70 \\
\hline $\mathrm{Cl}$ & 1.67 & $3.80 \times 10^{-4}$ & $1.49 \times 10^{-5}$ & 7.18 & 1.94 \\
\hline $\mathrm{Ar}$ & 0.45 & $2.08 \times 10^{-4}$ & $7.23 \times 10^{-6}$ & 6.87 & 0.72 \\
\hline $\mathrm{Ca}$ & 0.67 & $2.84 \times 10^{-4}$ & $9.82 \times 10^{-6}$ & 7.00 & 0.92 \\
\hline $\mathrm{Sc}$ & $\leq 1.79$ & $\leq 2.93 \times 10^{-6}$ & $\leq 1.00 \times 10^{-7}$ & $\leq 4.96$ & $\leq 2.11$ \\
\hline $\mathrm{Ti}$ & $\leq 1.02$ & $\leq 3.12 \times 10^{-5}$ & $\leq 1.00 \times 10^{-6}$ & $\leq 5.96$ & $\leq 1.31$ \\
\hline $\mathrm{V}$ & $\leq 2.06$ & $\leq 3.32 \times 10^{-5}$ & $\leq 1.00 \times 10^{-6}$ & $\leq 5.96$ & $\leq 2.33$ \\
\hline $\mathrm{Cr}$ & $\leq 1.33$ & $\leq 3.39 \times 10^{-4}$ & $\leq 1.00 \times 10^{-5}$ & $\leq 6.96$ & $\leq 1.62$ \\
\hline $\mathrm{Mn}$ & $\leq 0.53$ & $\leq 3.58 \times 10^{-5}$ & $\leq 1.00 \times 10^{-6}$ & $\leq 5.96$ & $\leq 0.83$ \\
\hline $\mathrm{Fe}$ & -0.24 & $6.92 \times 10^{-4}$ & $1.72 \times 10^{-5}$ & 7.24 & 0.00 \\
\hline Co & $\leq 2.02$ & $\leq 3.84 \times 10^{-4}$ & $\leq 1.00 \times 10^{-5}$ & $\leq 6.96$ & $\leq 2.27$ \\
\hline $\mathrm{Ni}$ & $\leq 0.75$ & $\leq 3.82 \times 10^{-4}$ & $\leq 1.00 \times 10^{-5}$ & $\leq 6.96$ & $\leq 1.04$ \\
\hline
\end{tabular}

Notes. $\quad{ }^{(a)}$ Bailer-Jones et al. (2018), (b) interpolated from evolutionary tracks of Dorman et al. (1993), (c) Schlafly \& Finkbeiner $(2011),{ }^{(d)}$ Bianchi et al. (2017), ${ }^{(e)}$ Zacharias et al. (2012), ${ }^{(f)}$ Kupfer et al. $(2015),{ }^{(g)}$ Cutri et al. (2003). 